ARTICLE

\title{
Synthetic biology based construction of biological activity-related library of fungal decalin-containing diterpenoid pyrones
}

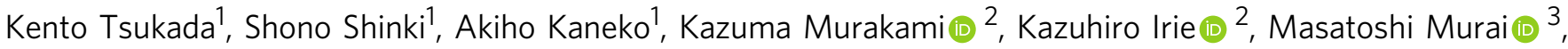
Hideto Miyoshi (10 ${ }^{3}$, Shingo Dan ${ }^{4}$, Kumi Kawaji ${ }^{5}$, Hironori Hayashi6, Eiichi N. Kodama ${ }^{5,6}$, Aki Hori ${ }^{7}$, Emil Salim7, Takayuki Kuraishi $^{7}$, Naoya Hirata $\mathbb{1}^{8}{ }^{8}$, Yasunari Kanda ${ }^{8}$ \& Teigo Asai (i) ${ }^{1,9 凶}$

A synthetic biology method based on heterologous biosynthesis coupled with genome mining is a promising approach for increasing the opportunities to rationally access natural product with novel structures and biological activities through total biosynthesis and combinatorial biosynthesis. Here, we demonstrate the advantage of the synthetic biology method to explore biological activity-related chemical space through the comprehensive heterologous biosynthesis of fungal decalin-containing diterpenoid pyrones (DDPs). Genome mining reveals putative DDP biosynthetic gene clusters distributed in five fungal genera. In addition, we design extended DDP pathways by combinatorial biosynthesis. In total, ten DDP pathways, including five native pathways, four extended pathways and one shunt pathway, are heterologously reconstituted in a genetically tractable heterologous host, Aspergillus oryzae, resulting in the production of 22 DDPs, including 15 new analogues. We also demonstrate the advantage of expanding the diversity of DDPs to probe various bioactive molecules through a wide range of biological evaluations.

\footnotetext{
${ }^{1}$ Department of Life Sciences, Graduate School of Arts and Sciences, The University of Tokyo, Komaba, Meguro-ku, Tokyo 153-8902, Japan. ${ }^{2}$ Division of Food Science and Biotechnology, Graduate School of Agriculture, Kyoto University, Kyoto 606-8502, Japan. ${ }^{3}$ Division of Applied Life Sciences, Graduate School of Agriculture, Kyoto University, Kyoto 606-8502, Japan. ${ }^{4}$ Division of Molecular Pharmacology, Cancer Chemotherapy Center, Japanese Foundation for Cancer Research, 3-8-31 Ariake, Koto-ku, Tokyo 135-8550, Japan. ${ }^{5}$ Department of Infectious Diseases, International Research Institute of Disaster Science, Tohoku Medical Megabank Organization, Tohoku University, 2-1, Seiryocho, Aoba-ku, Sendai 980-0875, Japan. ${ }^{6}$ Department of Intelligent Network for Infection Control, Graduate School of Medicine, Tohoku University, 2-1, Seiryocho, Aoba-ku, Sendai 980-0875, Japan. ${ }^{7}$ Faculty of Pharmacy, Institute of Medical, Pharmaceutical and Health Sciences, Kanazawa University, Kanazawalshikawa, Japan. ${ }^{8}$ Division of Pharmacology, National Institute of Health Sciences, Tonomachi, Kawasaki-ku, Kawasaki, Kanagawa 210-9501, Japan. ${ }^{9}$ Graduate School of Pharmaceutical Sciences, Tohoku University, Aoba-yama, Aoba-ku, Sendai 980-8578, Japan. ${ }^{凶}$ email: teigo.asai.c8@tohoku.ac.jp
} 
$\mathrm{N}$ atural products are historically unparalleled successful sources for drug discovery ${ }^{1,2}$. During secondary metabolite biosynthesis in living organisms, natural products are formed in vivo through multi-step enzymatic reactions ${ }^{3,4}$. In other words, natural product structures are constructed and tailored in repeated interaction with proteins in cells. This biosynthetic manner provides natural product with drug-advantageous properties such as potential protein interactivity, water solubility, and membrane permeability ${ }^{5}$. For this reason, the natural product chemical space is highly relevant to biological space ${ }^{6}$. Therefore, the discovery of natural products with novel structures and biological activities is an important challenge in the pharmaceutical field. Natural products with valuable biological activities are recognized to contain biologically relevant privileged structures, 7,8 whose analogues and congeners serve as excellent guides to discover not only more potent bioactive compounds but also various additional biological activities ${ }^{9,10}$. Thus, expanding the chemical space around biologically important natural products may accelerate drug discovery research ${ }^{11}$. Chemical synthesis, including divergent and diverted total syntheses, is a powerful strategy to produce bioactive natural product analogues and congeners ${ }^{12,13}$. A late-stage modification strategy is also a beneficial tool to generate natural product derivatives, such as diversity-oriented synthesis using natural products and their intermediate starting points ${ }^{14-17}$. However, the structural complexity and limited availability of natural products remain obstacles to synthesizing a large collection of natural products and their structural analogues in sufficient amounts. Thus, the development of a method that enables rapid and rational access to the chemical space around bioactive natural products and a reliable supply is required for drug discovery.

Fungi are among the most important microbial resources for drug discovery because of their ability to produce structurally diverse and biologically important natural products ${ }^{18}$. It is also well known that fungi possess extraordinary biosynthetic gene clusters that may encode highly diverse natural product biosynthetic pathways, and biosynthetic gene information has been accumulating at a rapidly accelerating rate in the past a decade because of genome sequencing innovation ${ }^{19}$. However, most biosynthetic pathways distributed in fungal genomes have not been linked with structural information ${ }^{20}$. This implies the presence of a large number of novel natural products that may be produced via those untapped pathways. A synthetic biology method based on heterologous biosynthesis coupled with genome mining is a promising approach to translate enormous amounts of biosynthetic gene information to richly diverse natural products. Genome mining based on biosynthetic studies has enabled rapid access to biosynthetic pathways not only for a target natural product and its related analogues but also for unexplored natural products. Heterologous biosynthesis has enabled to access compounds encoded by biosynthetic pathways found by genome mining and a number of total biosynthesis of natural products and discovering novel natural products have been reported ${ }^{21-28}$. In addition, combinatorial biosynthesis approach is powerful tool to generate non-natural analogues, which may be a great advantage to construct pharmaceutical beneficial screening library $29-32$. Thus, we apply the synthetic biology approach for rationally expanding the chemical space around a target natural product as follows (Fig. 1). The genome mining and reconstruction of a biosynthetic pathway for a target natural product in a heterologous host achieves its total biosynthesis, and the biosynthetic information allows us to mine its related biosynthetic pathways and access its analogues. We, then, apply pathway extension for combinatorial biosynthesis that can produce new natural product analogues that are not programmed in nature and more highly modified than programmed natural products.

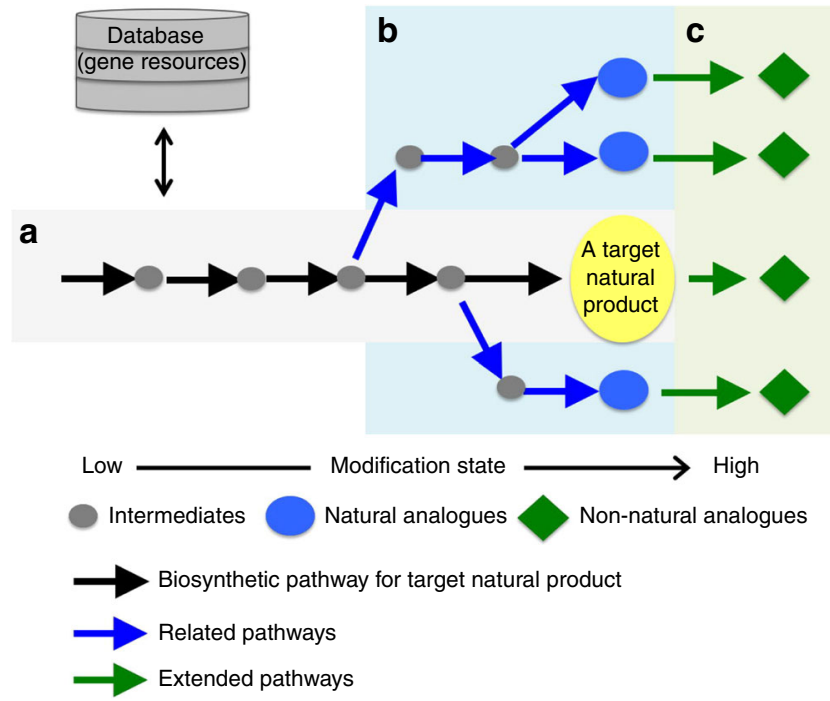

Fig. 1 Strategy for expanding the chemical space of a target bioactive natural product via a synthetic biology approach. a Genome mining and reconstruction of the target natural product biosynthetic pathway. b Genome mining and reconstruction of the related pathways to produce natural analogues. c Pathway extension for combinatorial biosynthesis by adding additional enzymes to the native pathways to produce non-natural analogues (additionally modified analogues), Grey circles, blue circles and green diamonds show intermediates, natural analogues and non-natural analogues, respectively. Black, blue and green arrows show biosynthetic pathway for target natural product, its related pathways and extended pathways, respectively.

Fungi produce decalin-containing diterpenoid pyrones (DDPs), a type of meroterpenoid natural products composed of a diterpenoid-derived decalin ring system linked to pyrone biosynthesized via polyketide pathways ${ }^{3}$. DDPs are found only in fungi, and over 20 DDPs have been reported to date (Supplementary Fig. 1). Interestingly, even small structural differences in each DDP show a wide range of biological activities, such as antiproliferative activity against cancer cell lines and immunosuppressive activity, implying that DDPs contain a privileged structure (Fig. 2) $33-41$. Thus, the chemical space of DDPs is likely relevant to a diverse biological space and expanding this space leads to the development of a valuable screening library for drug discovery. In addition to promising biological activities, the structural features of DDPs render them exciting targets for total synthesis ${ }^{42-44}$. Recently, excellent divergent total synthesis has been reported, which enables access to four DDPs via a common intermediate in less than twenty chemical reaction steps ${ }^{45}$. Nevertheless, the synthesis of diverse DDPs as well as non-natural analogues is a rather elaborate effort; and therefore, it is difficult to prepare a large collection of DDPs and expand their chemical space by chemical means.

Here, we demonstrate the advantage of the synthetic biology approach based on heterologous and combinatorial biosynthesis coupled with genome mining for constructing a biologically relevant DDP-focused library composed of a variety of DDPs. Although biosynthetic studies on DDPs are limited, a putative biosynthetic gene cluster and pathway for subglutinols $(s u b A-F)$ in an entomopathogenic fungus, Metarhizium robertsii, has been estimated (Fig. 3a). In addition, the functions of a non-reducing polyketide synthase (NR-PKS) SubA, geranylgeranyl diphosphate synthase (GGPPS) SubD and prenyltransferase (PT) SubC have been identified by a heterologous expression study ${ }^{46}$. We perform genome mining based on this information and find putative DDP gene clusters distributed in five fungal genera Arthrinium, 


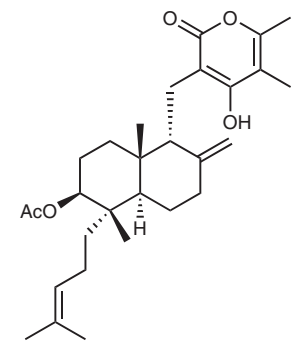

Sesquicillin A

(Acremonium sp.)

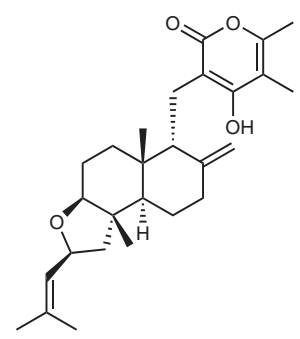

Subglutinol A

(Fusarium subglutinans)

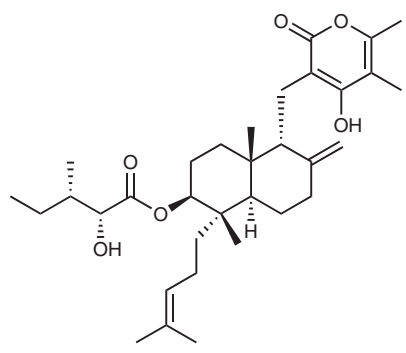

Metarhizin A (Metarhizium flavoviride)

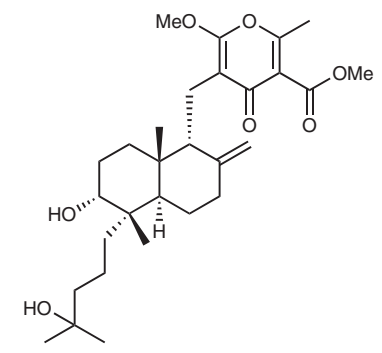

Colletotrichin

(Colletotrichum nicotianae)
Fig. 2 Examples of fungal decalin-containing diterpenoid pyrones (DDPs). DDPs have two privileged structures, pyrone (PKS) and decalin (terpenoid), and show a wide range of biological activities.

Metarhizium, Colletotrichum, Macrophomina and Fusarium fungi (Fig. 3b). According to bioinformatics analyses, we design five native pathways from those biosynthetic gene clusters and reconstruct them in Aspergillus oryzae NSAR1 $22-27,47$, an excellent heterologous host for the production of fungal natural products, to give intermediates and end products encoded in all the pathways. Subsequently, we conduct pathway extension for combinatorial biosynthesis by adding additional modification enzymes to the native DDP pathways, yielding unnatural DDP analogues. Overall, we successfully produce 22 DDPs including 15 analogues that have not been reported, which include intermediates, end products and additionally modified analogues. Because they all can be easily re-supplied by cultivation of the corresponding transformant, we are able to evaluate a variety of biological activities of the DDP-focused library and find wide range of potent bioactivities, such as cell cytotoxicity against cancer cell lines through mitochondrial complex III inhibition, antiproliferative activity against cancer stem-like cells, anti-HIV, preventing amyloid $\beta(\mathrm{A} \beta)$ aggregation in nucleation phase, paralysing activity against adult Drosophila and suppressing insect innate immune signal transduction. Most these biological activities firstly have been found in DDPs in this study.

\section{Results}

Genome mining and design of DDP biosynthetic pathways. To find biosynthetic gene clusters that may encode DDP pathways, we performed genome mining of the public databases and our original gene resources by using SubA (NR-PKS) as a query. As a result, five candidate gene clusters with $s u b A-E$ orthologous genes that may encode NR-PKS, GGPPS, PT, flavin adenine dinucleotide (FAD)-dependent epoxidase (FMOep) and terpene cyclase (TC) were found in five fungal genomes, Fusarium graminearum PH-1 (dpfgABCDEGHIJK), Macrophomina phaseolina MS6
(dpmpABCDEGHIJ) Colletotrichum higginsianum IMI349063 (dpchABCDEFGH), Metarhizium anisopliae E6 (dpmaABCDEF), and Arthrinium sacchari (dpasABCDEF), which we previously isolated from a spider (Fig. $3 \mathrm{~b}$ and Supplementary Table 1). The $d p m a$ gene cluster, which is identical to the sub gene cluster in $M$. robertsii, is widely conserved across the genus Metarhizium, and the $d p f g$ gene cluster is also broadly distributed in the genus Fusarium (Supplementary Fig. 2). To design native DDP biosynthetic pathways distributed in the five fungal genera, the five gene clusters were comparatively analysed based on amino acid sequence homology and reordered them as shown in Fig. $3 c$ (Supplementary Tables 2 and 3$)$. The five genes $(d p x x A B C D E)$ are highly conserved in each cluster, suggesting that all the pathways share the biosynthetic pathway for a common intermediate 4 . The differences in the genes at the tailoring steps in each pathway may diversify DDP biosynthesis. The three biosynthetic gene clusters in F. graminearum ( $d p f g$ cluster), M. phaseolina ( $d p m p$ cluster) and $C$. higginsianum (dpch cluster) contain two types of short chain dehydrogenase reductase (SDR) genes, $\operatorname{dpxxG}(\mathrm{SDR} 1)$ and $d p x x H$ (SDR2). Each of the $d p x x G$ and $d p x x H$ genes can be recognized as orthologous because of their high similarity (their encoded enzymes are approximately $60 \%$ identical each other); that is, each set of SDRs, $d p f g G H, d p m p G H$ and $d p c h G H$, may provide the same product from 4. Both the $d p f g$ and $d p m p$ clusters include an orthologous methyltransferase gene, $d p x x I$ (MT1), which it is absent in the $d p c h$ cluster, indicating that the $d p c h$ pathway is probably divided from the $d p f g$ and $d p m p$ pathways after SDR modification steps and that a remaining $d p c h F$ (FMO) would lead to the end product in the $d p c h$ pathway. Both the $d p m p$ and $d p f g$ clusters contain a P450 gene, $d p m p J$ and $d p f g J$, respectively, but they show low similarity to each other (their encoded enzymes are $\sim 20 \%$ identical to each other), suggesting that the $d p f g$ and $d p m p$ pathways may have branched after the MT1 modification step. Subsequently, $d p m p J$ and $d p f g J K$ afford the final products in each pathway. However, the $d p a s$ and dpma gene clusters possess only a modifying enzyme, FMO dpas $F$ and FAD-dependent BBE domain-containing oxidoreductase (BBE) dpmaF, respectively. Therefore, both pathways are likely branched at the initial tailoring stage, leading to the final products. As a result, we predicted the treelike native DDP pathways, as depicted in Fig. 3d. Considering previously reported DDPs and their producing fungi, the $d p c h$ and $d p m a$ pathways may produce higginsianin $\mathrm{A}^{40}$ and subglutinol $\mathrm{A}^{38,46}$, respectively. However, the orphan $d p f g, d p m p$ and $d p a s$ pathways may provide new DDP analogues.

Reconstitution of DDP pathways. We reconstituted all the pathways by stepwise introduction of the biosynthetic genes in a heterologous host $A$. oryzae NSAR1 according to the hypothetical biosynthetic pathways (Fig. 3d and Supplementary Fig. 5). Because it is difficult to obtain genome-sequenced strains of $F$. graminearum $\mathrm{PH}-1$, M. phaseolina MS6, C. higginsianum IMI349063 and M. anisopliae E6, we used F. graminearum 50218, M. phaseolina NBRC7317, C. higginsianum MAFF305635 and $M$. anisopliae NBRC103233 instead as gene donors.

Initially, we introduced $d p a s A C D$ into $A$. oryzae to construct the A. oryzae transformant with dpas $A C D$ ( $A O-d p a s A C D)$, which, as expected, produced prenylated $\left(\mathrm{C}_{20}\right)$ a-pyrone 2 (Fig. $3 \mathrm{a}$ and Supplementary Fig. 7). We subsequently introduced $d p a s B E$ into $A O$-dpas $A C D$ to construct $A O-d p a s A B C D E$, which provided the common intermediate $4\left(87 \mathrm{mg} \mathrm{L}^{-1}\right)$ (Supplementary Fig. 7), demonstrating its biosynthetic machinery for the first time. We also characterized $d p f g B E, d p m p B E$ or $d p c h B E$ as sharing the same function of $d p a s B E$ by introducing them into $A O-d p a s A C D$ (Supplementary Fig. 7). 
a

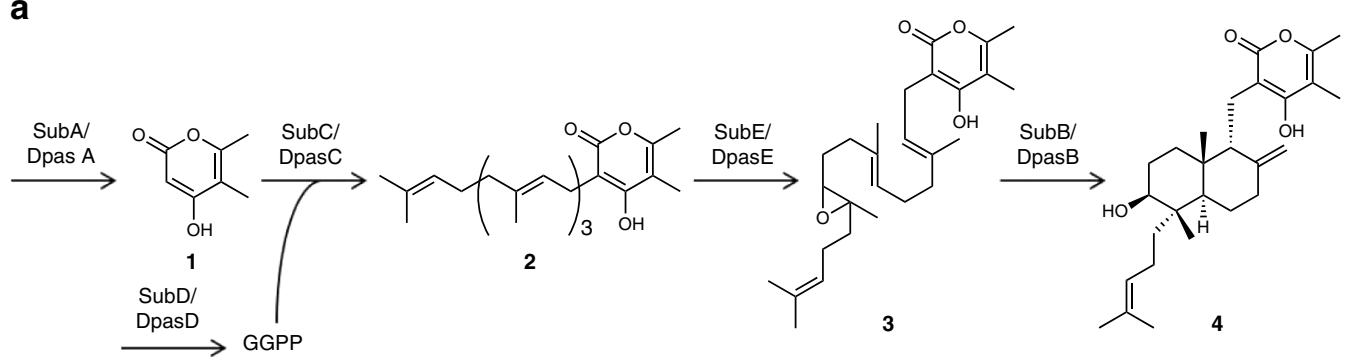

b

Fusarium graminearum (dpfg cluster)

Macrophomina phaseolina (dpmp cluster)
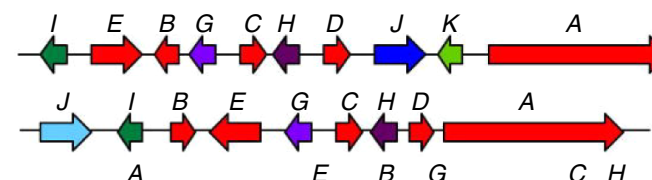

Colletotrichum higginsianum (dpch cluster)

Arthrinium sacchari (dpas cluster)

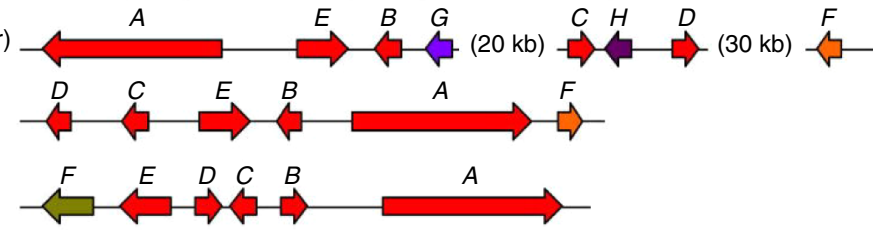

Metarhizium anisopliae (dpma cluster) $=M$. robertsii sub cluster

C

\begin{tabular}{|c|c|c|c|c|c|c|c|c|c|c|c|c|}
\hline & $\begin{array}{c}d p x x A(N R-P K S) \\
\text { (SAT-KS-AT-PT-ACP-MT) }\end{array}$ & $\begin{array}{c}d p x x D \\
\text { (GGPPS) }\end{array}$ & $\begin{array}{c}d p x x \mathrm{C} \\
\text { (PT) }\end{array}$ & $\begin{array}{c}\text { dpxxE } \\
\text { (FMOep) }\end{array}$ & $\begin{array}{l}d p x x B \\
\text { (TC) }\end{array}$ & $\begin{array}{l}d p x x G \\
\text { (SDR1) }\end{array}$ & $\begin{array}{l}d p x x \mathrm{H} \\
\text { (SDR2) }\end{array}$ & $\begin{array}{l}d p x x I \\
\text { (MT1) }\end{array}$ & $\begin{array}{l}d p x x J \\
(\mathrm{P} 450)\end{array}$ & $\begin{array}{l}d p x x K \\
\text { (MT2) }\end{array}$ & \multicolumn{2}{|c|}{$\begin{array}{c}d p x x F \\
\text { (BBE / FMO) }\end{array}$} \\
\hline$d p f g$ & & & $c$ & & $\Leftrightarrow$ & $\vec{z}$ & c & $\Rightarrow$ & & $\Rightarrow$ & & \\
\hline$d p m p$ & & & & & & & & $\Rightarrow$ & 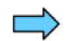 & & & \\
\hline$d p c h$ & & & $\Rightarrow$ & & & $\Rightarrow$ & & & & & & $\Rightarrow$ \\
\hline dpas & & & $\Rightarrow$ & & $\Rightarrow$ & & & & & & & $\Rightarrow$ \\
\hline dpma & & & $\rightarrow$ & & $\Rightarrow$ & & & & & & $\Rightarrow$ & \\
\hline $\begin{array}{l}\text { Tdentity } \\
\text { (a.a.) }\end{array}$ & $45-57 \%$ & $66-83 \%$ & $51-73 \%$ & $47-64 \%$ & $58-78 \%$ & $60-63 \%$ & $61-67 \%$ & $62 \%$ & $21 \%$ & & & $34 \%$ \\
\hline
\end{tabular}

d

Native pathways

Extended steps

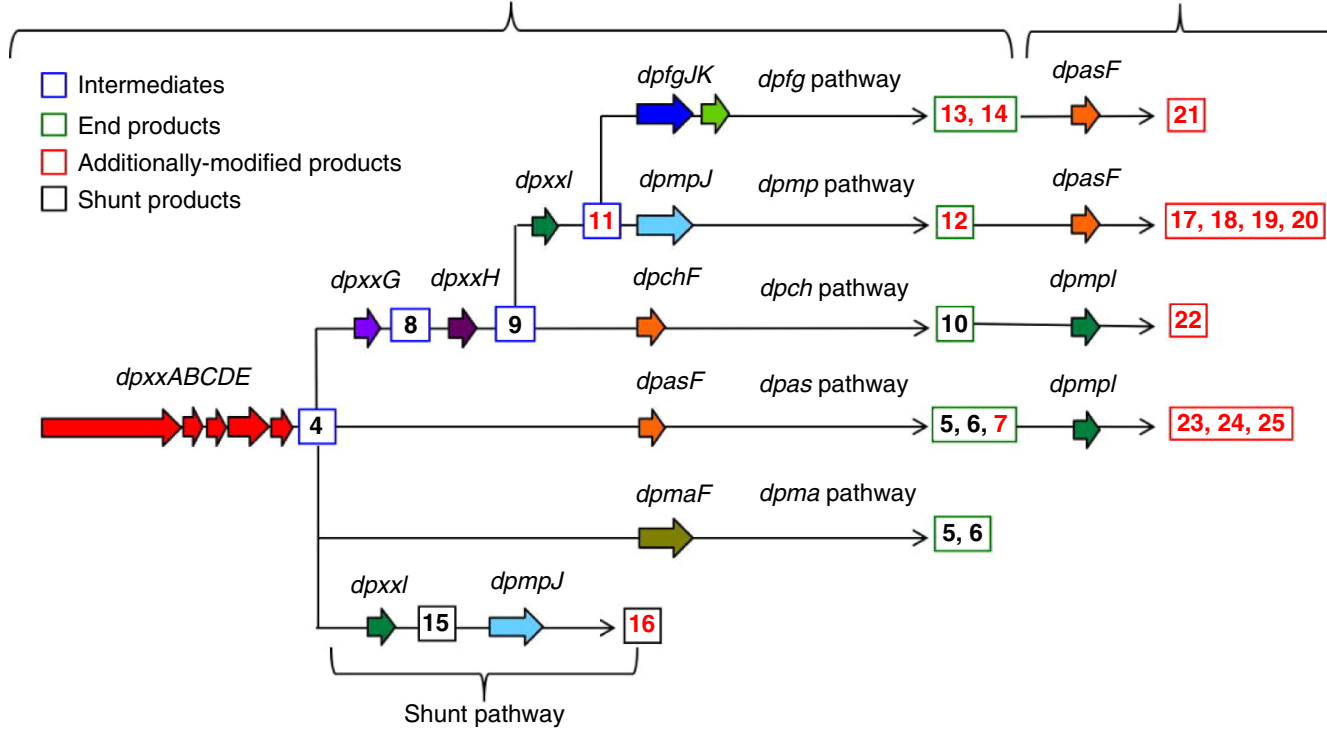

Fig. 3 Genome mining and design of DDP pathways. a Biosynthetic pathway for common intermediate 4. b DDP biosynthetic gene clusters distributed in five fungal genera. c Comparative analysis of each biosynthetic gene cluster. d Design of native pathways, extended steps and one shunt pathway heterologously reconstituted in this study and summary of products (red numbers show compounds that have not been reported.) produced through the DDP pathways (blue square, green square, red square and black square show intermediates, end products, additionally modified products and shunt products, respectively). 

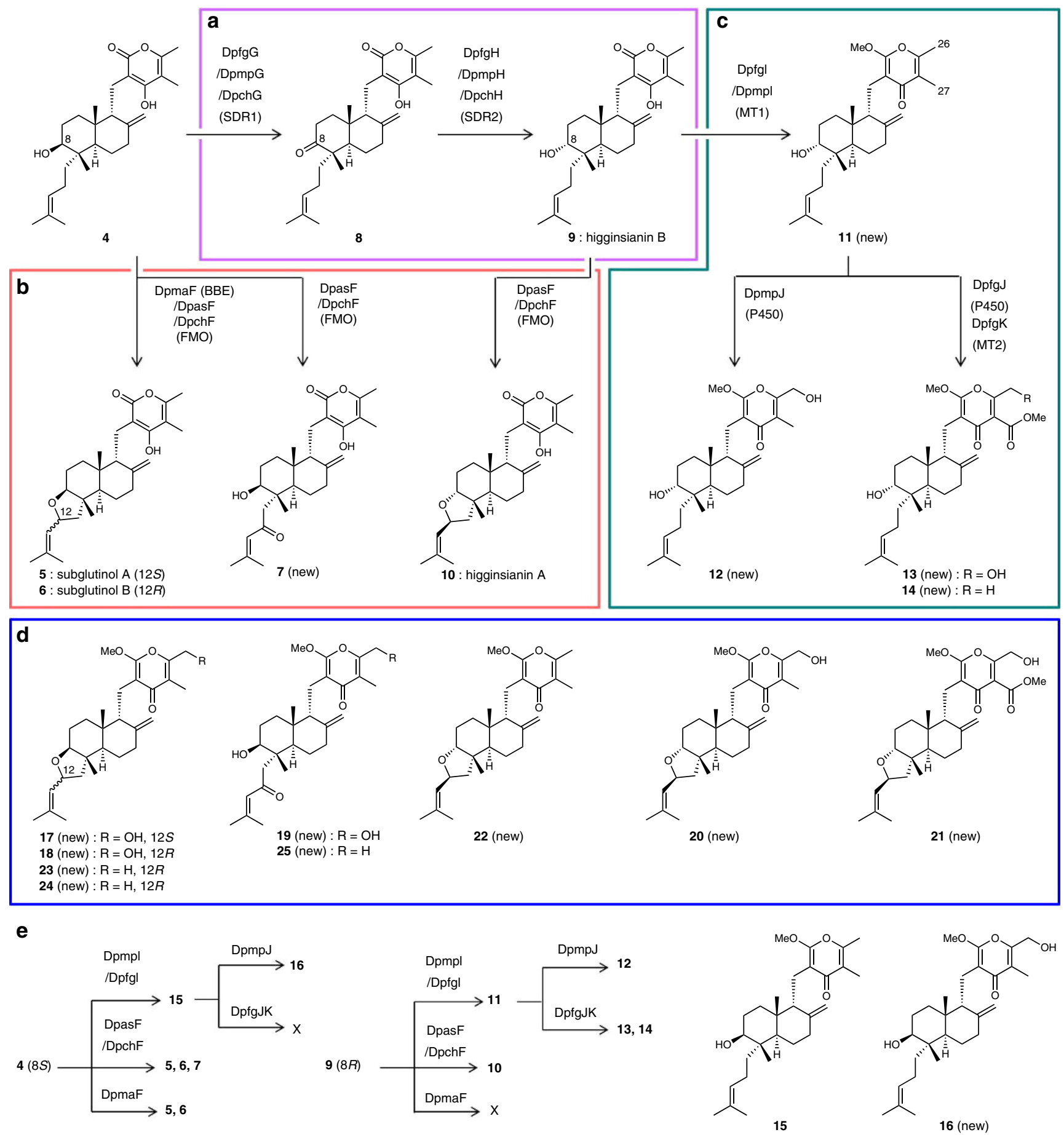

Fig. 4 Summary of enzymatic conversions in the DDP pathways. a C-8 stereochemistry inversion steps. b Modifications at the $C_{5}$ unit. $\mathbf{c}$ Modifications on the pyrone rings. $\mathbf{d}$ Structures of additionally modified DDPs produced through the extended pathways. e Summary of substrate selectivities of the modification steps in the DDP pathways.

Next, we aimed to comprehensively reconstitute modification steps in native DDP pathways by using the 4-producing transformants as a platform. We reconstituted the dpas and dpma pathways by introducing an FMO gene dpasF or a BBE gene dpmaF into $A O-d p a s A B C D E$ to give $A O-d p a s A B C D E F$ and AO-dpasABCDE-dpmaF. AO-dpasABCDE-dpmaF provided subglutinols $\mathrm{A}\left(5,89 \mathrm{mg} \mathrm{L}^{-1}\right)$ and $\mathrm{B}\left(6,6 \mathrm{mg} \mathrm{L}^{-1}\right)$ as previously suggested in the sub cluster ${ }^{46}$ (Supplementary Fig. 12). On the other hand, AO-dpasABCDEF produced a new DDP analogue with an enone system at the $\mathrm{C}_{5}$ unit $\left(7,6 \mathrm{mg} \mathrm{L}^{-1}\right)$ as well as subglutinols A $\left(5,62 \mathrm{mg} \mathrm{L}^{-1}\right)$ and $\mathrm{B}\left(\mathbf{6}, 5 \mathrm{mg} \mathrm{L}^{-1}\right)$ (Supplementary Figs. 12 and 13). These results suggested that both DpasF and DpmaF are involved in tetrahydrofuran (THF) ring formation at the $\mathrm{C}_{5}$ unit, while DpasF possesses an additional catalytic ability of multi-step oxidations to generate the enone at the $\mathrm{C}_{5}$ unit in 7 (Fig. 4b).

We then aimed to reconstitute the $d p c h, d p m p$ and $d p f g$ pathways in $A$. oryzae. The introduction of $d p m p G$ or dpmpGH into $A O-d p a s A B C D E$ afforded two transformants, AO-dpasABCDE$d p m p G$ and AO-dpasABCDE-dpmpGH. AO-dpasABCDE-dpmpG 
accumulated ketone $8\left(23 \mathrm{mg} \mathrm{L}^{-1}\right)$, while $A O$-dpasABCDE$d p m p G H$ gave higginsianin $\mathrm{B}\left(\mathbf{9}, 28 \mathrm{mg} \mathrm{L}^{-1}\right)$ (Supplementary Fig. 17). This result showed that SDR1, DpmpG, oxidized the $8 S$ hydroxy group to a ketone and SDR2, DpmpH, reduced the ketone to the $8 R$ hydroxy group in a similar manner to that in andrastin biosynthesis $^{48}$ (Fig. 4a). We also demonstrated that $d p f g G H$ and $d p c h G H$ were involved in the same inversion of the stereochemistry at C-8 (Supplementary Fig. 17). The entire $d p c h$ pathway was heterologously reconstituted by introducing $d p c h G H F$ into a 4producing transformant, and the resulting transformant, as expected, produced higginsianin A $\left(\mathbf{1 0}, 28 \mathrm{mg} \mathrm{L}^{-1}\right)$ (Fig. $4 \mathrm{~b}$ and Supplementary Fig. 21). We reconstituted whole $d p m p$ and $d p f g$ pathways by using higginsianin B (9) producing transformants as a platform. The introduction of $d p m p I$ and $d p f g I$ into the platforms yielded $A O-d p a s A B C D E-d p m p G H I$ and $A O-d p a s A B C D E-d p f g G H I$, both of which produced a new intermediate $11\left(29 \mathrm{mg} \mathrm{L}^{-1}\right.$ and 20 $\mathrm{mgL}^{-1}$, respectively). Moreover, $d p m p I J$ and $d p f g I J K$ were introduced to construct $A O-d p a s A B C D E-d p m p G H I J$ and $A O$ $d p a s A B C D E-d p f g G H I J K$. The transformant expressing the whole dpmp gene cluster, AO-dpasABCDE-dpmpGHIJ, afforded a new DDP with a C-26 primary alcohol on the $\gamma$-pyrone moiety (12, $21 \mathrm{mg} \mathrm{L}^{-1}$ ) (Fig. 4c and Supplementary Fig. 25). On the other hand, the transformant expressing the whole $d p f g$ pathway, $A O$ $d p a s A B C D E-d p f g G H I J K$, produced the new DDPs 13 (major product, $5 \mathrm{mg} \mathrm{L}^{-1}$ ) and $\mathbf{1 4}$ (minor product, $2 \mathrm{mg} \mathrm{L}^{-1}$ ) with a highly oxidized $\gamma$-pyrone moiety including a methyl ester (Fig. 4c and Supplementary Fig. 25), like colletotrichin obtained from $C$. nicotianae ${ }^{41}$. The results suggested that a P450, DpmpJ, oxidized C26 methyl to primary alcohol, DpfgJ, catalysed a three-step oxidation at C-27 to generate a carboxylic acid as well as C-26 hydroxylation. The results also indicated that an MT1, DpfgI, is involved in the same methylation as DpmpI, while an MT2, DpfgK, methylates the carboxylic acid generated by DpfgJ. We thus completely reconstructed all the DPP native pathways distributed in five fungi, resulting in the production of 11 DDPs, including five new analogues. As expected, the $d p m a$ and $d p c h$ pathways encoded the biosynthetic pathways for subglutinols $(5,6)$ and higginsianin A (10), respectively, and the orphan $d p a s, d p m p$ and $d p f g$ pathways provided new DDP analogues.

We also investigated substrate selectivities of the modification enzymes using $A$. oryzae heterologous expression system. In summary, DpmaF and DpfgJ strictly recognized the C-8 configuration, while DpasF, DpchF, DpmpI, DpfgI and DpmpJ showed tolerant substrate selectivity, which became an advantage for the generating diversity in combinatorial biosynthesis. Through the experiments, we found and reconstituted a shunt DDP pathway in $A$. oryzae, which afforded viridoxin A hydrolysate $15^{33}\left(42 \mathrm{mg} \mathrm{L}^{-1}\right)$ and a new DDP analogue 16 $\left(6 \mathrm{mg} \mathrm{L}^{-1}\right)$ (Figs. 3d, 4e, Supplementary Figs. 29, 31 and Supplementary Note 15).

Pathway extension for combinatorial biosynthesis. The reactions of each enzyme in all the pathways are summarized in Fig. $4 \mathrm{a}-\mathrm{c}$. The enzymes that catalysed $\mathrm{C}_{5}$ unit modifications were specifically distributed in the dpma, dpas and $d p c h$ pathways, while the enzymes involved in the pyrone moiety modifications were localized in the other pathways. However, no pathway that containing both $\mathrm{C}_{5}$ unit and pyrone moiety modification enzymes is encoded in native DDP biosynthetic gene clusters. Therefore, we aimed to generate unnatural DDPs with further modified structures than those of the end products in each DDP pathway and conducted combinatorial biosynthesis by combining $\mathrm{C}_{5}$ unitmodifying pathways with pyrone-decorating pathways (Fig. 3d). We chose $d$ pas $F$ among $C_{5}$ unit-modifying enzymes as an additional modification enzyme, because DpasF (FMO) enables the generation of four types of $\mathrm{C}_{5}$ unit moieties through its multifunctional oxidative ability, and we designed two extended pathways by adding $d p a s F$ to the $d p m p$ and $d p f g$ pathways. In addition, MT1 (dpmpI) was connected to the dpas and $d p c h$ pathways. Thus, we designed and reconstituted the four extended pathways in A. oryzae (Fig. $3 \mathrm{~d}$ ). One transformant ( $1 p m p$ pathway $+d p a s F)$, as expected, produced four new analogues, 17 $\left(5 \mathrm{mg} \mathrm{L}^{-1}\right), 18\left(3 \mathrm{mg} \mathrm{L}^{-1}\right), 19\left(16 \mathrm{mg} \mathrm{L}^{-1}\right)$, and $20\left(5 \mathrm{mg} \mathrm{L}^{-1}\right)$, due to the promiscuity of DpmpJ (Figs. $3 \mathrm{~d}$, $4 \mathrm{~d}$ and Supplementary Fig. 35). Another transformant ( $d p f g$ pathway $+d p a s F)$ afforded only the new analogue $21\left(6 \mathrm{mg} \mathrm{L}^{-1}\right)$, the most modified compound in this study because DpfgJ (P450) strictly recognizes C-8 stereochemistry (Figs. 3d, 4d and Supplementary Fig. 36). In this transformant, another expected product originating from 14 could not be observed in HPLC analysis. The third transformant $(d p c h$ pathway $+d p m p I)$, as expected, produced $O$-methylated higginsianin $\mathrm{A}\left(\mathbf{2 2}, 17 \mathrm{mg} \mathrm{L}^{-1}\right)$, and the fourth transformant (dpas pathway $+d p m p I)$ provided $23\left(23 \mathrm{mg} \mathrm{L}^{-1}\right), 24\left(7 \mathrm{mg} \mathrm{L}^{-1}\right)$ and $25\left(3 \mathrm{mg} \mathrm{L}^{-1}\right)$ (Figs. 3d, $4 \mathrm{~d}$ and Supplementary Fig. 40). As expected, all the non-natural analogues produced through combinatorial biosynthesis contained modifications on both the $\mathrm{C}_{5}$ unit and the pyrone moiety. Since the four re-designed extended pathways are not found in the genome databases, of course, every compound produced through the pathways had new structures.

Thus, we achieved the comprehensive production of fungal DDPs through the reconstitution of five-native pathways, one shunt pathway and four extended pathways in A. oryzae and produced 22 DDPs, including 15 new compounds. Among these 22 compounds, 11 compounds came from native pathways, 2 compounds were biosynthesized through shunt pathways, and 9 compounds were produced via extended pathways (Supplementary Fig. 43, Supplementary Table 19). The new compounds, 7, 11-14 and 16-25 were named as FDDP A-O, respectively. All the compounds produced in this study were purified, and their structures were fully determined by spectral analyses. The absolute configuration of the common intermediate (4) was determined by the modified Mosher's method ${ }^{49}$ (Supplementary Fig. 10), while those of 5, 6, 9 and 10 were identified by comparing their optical rotation with reported values ${ }^{40,43}$. The absolute configurations of the other DDPs were determined based on their biosynthetic relationships. The titres of all the DDPs based on HPLC analysis of all the transformants are summarized in Supplementary Table 19.

Antiproliferative effects on cancer stem-like cells. Initially, we evaluated new DDP analogues for their antiproliferative activities across the panel of 39 human cancer cell lines, JFCR3950,51. The assay not only showed their cytotoxic effects but also provided the characteristic profiles similar to those of antimycin A and myxothiazol, known inhibitor of mitochondrial complex III (Supplementary Figs. 44, 45 and Supplementary Tables 21, 22). We then revealed that most DDPs, except for enone-containing compounds, selectively prevented mitochondrial complex III, and the effect was the same degree as that of antimycin A in vitro assay (Supplementary Table 20).

We also evaluated antiproliferative activity against cancer stem cells (CSCs), which are a small sub-population in tumour bulk identified in most cancer cells and clinical samples ${ }^{52}$. CSCs have been a major problem in cancer therapy because they are responsible for recurrence, metastasis and drug resistance to chemotherapeutic agents that affect proliferative cells ${ }^{53-56}$. AntiCSC activity was evaluated by the inhibition of sphere-forming ability, a well-studied method to enrich the CSC-like population, and selective cytotoxicity against one of the most reliable CSC 
a

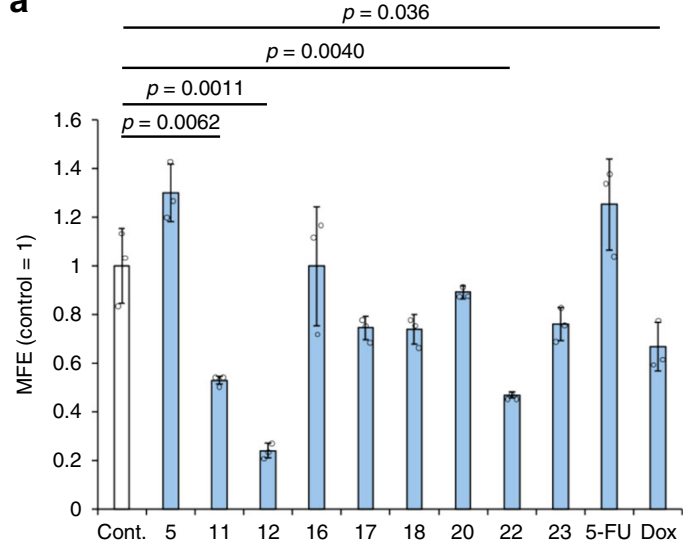

C

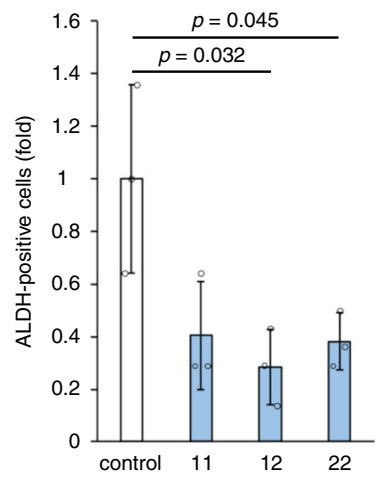

d

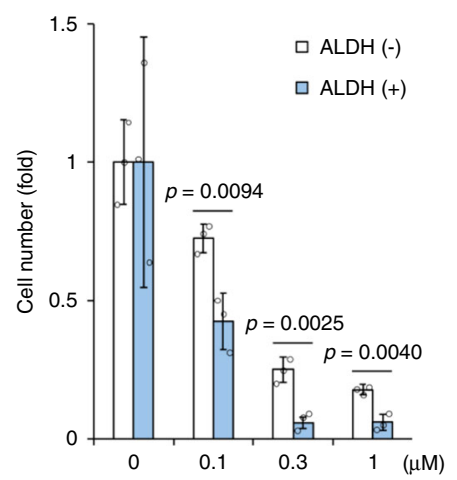

b

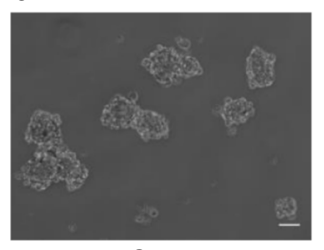

Cont.

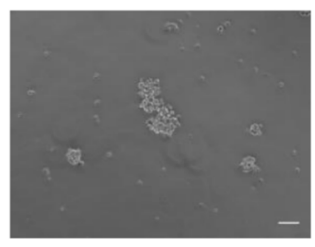

11

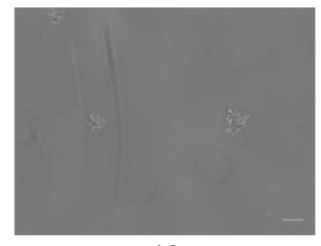

12

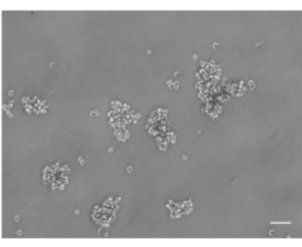

16

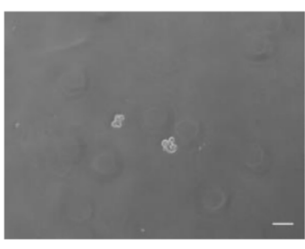

22

Fig. 5 DDPs show anti-CSC activity in breast cancer cell line MCF-7. a Effect of DDPs on mammosphere formation. After the cells were cultured in the presence of $5 \mu \mathrm{M}$ DDPs for 7 days, the number of mammospheres was microscopically counted and the percentage of mammosphere-forming cells was determined as mammosphere-forming efficiency (MFE; \%). Data represent mean \pm s.d. from three independent replicate experiments. $P$-values were calculated by a two-sided unpaired Student's $t$-test, compared with control. b Micrographs show representative images of mammosphere formation from three independent replicate experiments using compound 11, 12, 16, 22. The scale bar, $50 \mathrm{~mm}$. c Effect of DDPs ( $1 \mu \mathrm{M}, 3$ days) on ALDH-positive cells in MCF-7 cells. Data represent mean \pm s.d. from three independent replicate experiments. $P$-values were calculated by a two-sided unpaired Student's $t$-test, compared with control. d Dose-dependent effect of compound $\mathbf{1 2}$ on ALDH-positive cells in MCF-7 cells. Data represent mean \pm s.d. from three independent replicate experiments. $P$-values were calculated by a two-sided unpaired Student's $t$-test. Source data underlying Fig. $5 a$, $c, d$ are provided in a Source Data file.

markers, aldehyde dehydrogenase (ALDH)-positive cells ${ }^{55}$. We selected compounds $5,11,12,17,18,20,22$ and 23 according to dose-dependent cytotoxicity against the bulk of MCF-7 cells for the mammosphere formation assay (Supplementary Fig. 46a). Low-toxicity 16 was also added as a negative control. Each compound was tested at $5 \mu \mathrm{M}$, and 11, 12 and 22 clearly inhibited sphere formation (Fig. 5a, b) more effectively than the anticancer drugs 5-fluorouracil (5-FU) and doxorubicin. Interestingly, 12 showed the strongest effect on sphere formation, while its C-8 epimer 16 did not affect sphere formation, suggesting that the $8 R$ configuration significantly contributed to this activity. We next evaluated the three DDPs that inhibited mammosphere formations against ALDH-positive cells, a functional marker for breast CSCs. MCF-7 cells were treated with $1 \mu \mathrm{M}$ of 11,12 or 22 , and the ALDH-positive cell population was analysed by using a fluorescence activated cell sorter (Supplementary Fig. 46b). The results showed that 11,12 and 22 reduced the population of ALDH-positive cells (Fig. 5c). The most effective compound, 12, was tested at lower concentrations and showed selective and potent cytotoxicity against ALDH-positive cells (Fig. 5d). Thus, for the first time, we successfully found DDPs with potent antiproliferative effects on the CSC-like population in MCF-7 cells, which may lead to new anti-CSC drugs. Interestingly, subtle structural differences in DDPs generated the difference in antiCSC activity.

Biological activity against Drosophila. We screened the DDPs on the Drosophila assay system for cytotoxicity, inhibitory activity of innate immune signalling and insecticidal activity ${ }^{57}$. The cytotoxicity of the DDPs was evaluated using two cell lines, embryonic macrophage-derived DL1 cells ${ }^{58-60}$ and larval blood cell-derived $1(2) \mathrm{mbn}$ cells ${ }^{61}$, and their $\mathrm{IC}_{50}$ values are listed in Supplementary Table 23. Most DDPs except for compounds with the enone at the $C_{5}$ unit $(7,19$ and 25$)$ showed potent antiproliferative activity, and they affected DL1 cell lines more potently. Notably, subglutinol A (5) and 21 strongly inhibited cell growth in the DL-1 cell line ( $\mathrm{IC}_{50}=12 \mathrm{nM}$ (5) and $15 \mathrm{nM}(\mathbf{2 1})$ ).

We next tested the ability of the DDPs to affect the innate immune pathways, namely, the Toll and immune deficiency (IMD) pathways, which are the front line of defence against infection by microorganisms ${ }^{57}$. No compound showed a remarkable effect on the Toll pathway, while $\mathbf{2 1}$ inhibited the IMD pathway with an $\mathrm{IC}_{50}$ of $0.27 \pm 0.01 \mu \mathrm{M}$ (Fig. 6a). This result implies that the $\gamma$-pyrone moiety that is observed in 21 might have a role in the activity and that the THF ring at the $\mathrm{C}_{5}$ unit enhances the effect, since $\mathbf{1 3}$ tend to show a moderate inhibitory effect on the IMD pathway. Then, we evaluated the insecticidal activity of the most potent cytotoxic $\mathbf{5}$ and IMD pathway inhibitor 21 by using adult Drosophila (Fig. 6b). The result clearly indicates that 5 efficiently paralysed adult Drosophila $1 \mathrm{~h}$ after injection, implying that subglutinol A (5) is one of the virulence factors of entomopathogenic Metarhizium fungi against insects. Thus, we successfully found unique biological activity against Drosophila in $\mathbf{5}$ and $\mathbf{2 1}$, which may potentially be developed as insecticides.

Anti-HIV assay. We examined the inhibitory activities of DDPs against various species of bacteria (both gram-negative and grampositive bacteria), fungi and viruses. No DDPs showed significant anti-bacterial or anti-fungal activities. In contrast, preliminary 

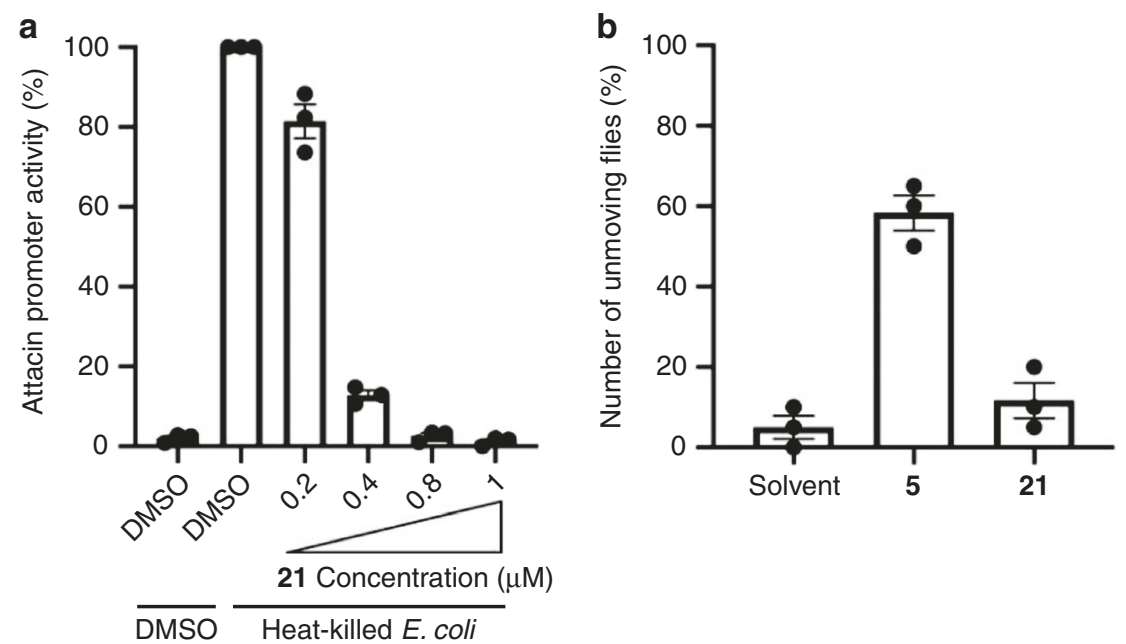

Fig. 6 Biological activity against Drosophila. a Inhibitory effect of compound $\mathbf{2 1}$ on the IMD pathway Drosophila I(2)mbn cells were stimulated with heatkilled E. coli and the activity of the Attacin promoter, a read-out of the activation of the IMD pathway, was monitored by a luciferase assay. Data are means \pm SEM of triplicate wells from a single experiment and are representative of two independent experiments. $\mathbf{b}$ Toxic effect of compound $\mathbf{5}$ on Drosophila adult. Adult flies (c.a. $1 \mathrm{mg}$ each) were injected with the indicated compounds ( $3.5 \mathrm{ng}$ each), and the flies that did not show movement or only faint shaking of their legs were counted as unmoving flies. Data are the means \pm SEM of triplicate samples from a single experiment and are representative of two independent experiments. Source data underlying Fig. 6a, b are provided in a Source Data file.

screening of the DDPs for anti-viral agents with hepatitis B virus (HBV), measles virus (MV), Epstein-Barr virus (EBV), herpes simplex virus (HSV) and human immunodeficiency virus-1 (HIV1) indicated their selective inhibition of HIV. We further investigated the anti-HIV activity of the DDPs by the MAGI assay 62 using $\mathrm{AZT}$ as positive control $\left(\mathrm{EC}_{50}=29.7 \mathrm{nM}\right.$ and $\mathrm{CC}_{50}>$ $10000 \mathrm{nM})$. Some of compounds, such as $5,10,11,12,14,17,18$, 20, 23 and 24, exerted specific anti-HIV activity $\left(\mathrm{EC}_{50}<200 \mathrm{nM}\right)$ without affecting other viruses (Supplementary Table 24). Among them, 20 showed the strongest anti-HIV activity without apparent cytotoxicity $\left(\mathrm{EC}_{50}=8.5 \mathrm{nM}\right.$ and $\left.\mathrm{CC}_{50}>10,000 \mathrm{nM}\right)$. Other compounds also showed moderate to substantial activity but the effective concentrations were high; therefore, their toxicity made it difficult to clearly distinguish their anti-HIV effect.

Screening of amyloid $A \boldsymbol{\beta} 42$ aggregation inhibitors. The aggregation of the 42 -mer-amyloid $\beta(A \beta 42)$ is involved in the pathogenesis of Alzheimer's disease $(\mathrm{AD})^{63}$. A nucleationdependent polymerization model that is composed of nucleation and elongation phases is generally accepted as the aggregation mechanism of $A \beta 42^{64}$. In the nucleation phase, the monomer of A $\beta 42$ gradually forms low-molecular-weight oligomers (called nuclei), which cause synaptotoxicity and memory loss ${ }^{65}$.

We evaluated the ability of DDPs to inhibit A $\beta 42$ aggregation by using the Th-T (thioflavin-T) assay, which is a conventional and useful method for quantifying $\mathrm{A} \beta$ aggregates (Supplementary Fig. 47). Consideration of the preliminary results and SARs, we picked up 12, 13, 14, 20, and 21, and tested them again (Fig. 7a). The fluorescence of $\mathrm{A} \beta 42$ and the Th-T complex began to increase after $4 \mathrm{~h}$ of incubation, and only 13 and 21 delayed the nucleation phase of $A \beta 42$ until 6 and $13 \mathrm{~h}$, respectively. In contrast, the congeners of $\mathbf{1 3}$ and $\mathbf{2 1}$ regarding the primary alcohol or methyl ester group, such as 12,14 and 20 showed no delay even though 12, 14 and 20 partly suppressed the elongation of $A \beta 42$. In TEM analysis, the formation of typical amyloid fibrils of $A \beta 42$ was strongly prevented only by 13 and 21, leading to the fragmentation of fibrils (Fig. 7b).

To further analyse the inhibitory mechanism, we subjected a mixture of $\mathrm{A} \beta 42$ and 21 after $1 \mathrm{~h}$ of incubation at room temperature to liquid chromatography/quadrupole time-of-flight mass spectrometry (LC/Q Tof-MS). The possible peaks of A $\beta 42-$ 21 adducts were detected, and then the mass envelopes at $+7,+6$, +5 , and +4 charge distribution corresponded to the Michael adduct based on the loss of $32 \mathrm{Da}$ (Supplementary Figs. 48b-d, 49). However, no such adduct was found in the presence of 20 as a negative control (Supplementary Fig. 48a). To identify the specific amino acids involved in the interaction of $\mathbf{2 1}$ with the $A \beta$ monomer, we performed LC-MS/MS analysis with collisioninduced dissociation (CID) using E22P, M35ox-A $\beta 9-35$ as a toxic conformer surrogate ${ }^{66,67}$. Based on a large number of fragmented b ions and y ions, Lys16 in A $\beta 42$ (DAEFRHDSGYEVHHQKLVFFAEDVGSNKGAIIGLMVGGVVIA) seemed to be one of the target amino acid residues of 21 (Supplementary Fig. 50).

To investigate the reaction mechanism between the highly oxidized $\gamma$-pyrone observed in $\mathbf{1 3}$ and $\mathbf{2 1}$, and the lysine residue in $\mathrm{A} \beta 42$ with a decrease of $32 \mathrm{Da}$, we carried out the following experiment. Benzyl-protected lysine 28 was reacted with 13 and $\mathbf{2 1}$ as well as the structurally related $\mathbf{1 4}$ and $\mathbf{2 0}$ in THF at room temperature (Supplementary Note 30 ), and each reaction was monitored by HPLC analysis at $1 \mathrm{~h}$ and $10 \mathrm{~h}$ after mixing (Supplementary Fig. 51a). In the reaction of 13, two new peaks gradually appeared at $1 \mathrm{~h}$ later, and $\mathbf{1 3}$ completely converted to the new peaks 26 and 27 (Supplementary Fig. 51b) in 10 h. LCMS analysis showed the same molecular ion peak of 26 and 27 at $\mathrm{m} / z$ $863[\mathrm{M}+\mathrm{Na}]^{+}$, which suggested that 13 connected to 28 with a decreasing of $32 \mathrm{Da}$ in the same situation described above. The reaction of $\mathbf{2 1}$ proceeded in the same manner as that of 13, whereas 14 and 20 did not afford any adducts under the same reaction conditions (Supplementary Fig. 51), suggesting that the $\gamma$-pyrone structure, including a hydroxyl group and carboxylic methyl ester, was necessary for the reaction. After scaling up the reaction, we isolated $\mathbf{2 6}$ and $\mathbf{2 7}$, which are in the C-21 epimer relationship, and determined their structures (Fig. 7c, Supplementary Note 31). From their structures, we proposed the following reaction mechanism: 13 took the ortho ester form, which increased electrophilicity at C-24, and the amine in lysine performed a nucleophilic attack at C-24 with elimination of the methoxy group followed by electron transfer to form 26 and 27 (Fig. 7c and Supplementary Fig. 52). However, the pyrone moiety 
a

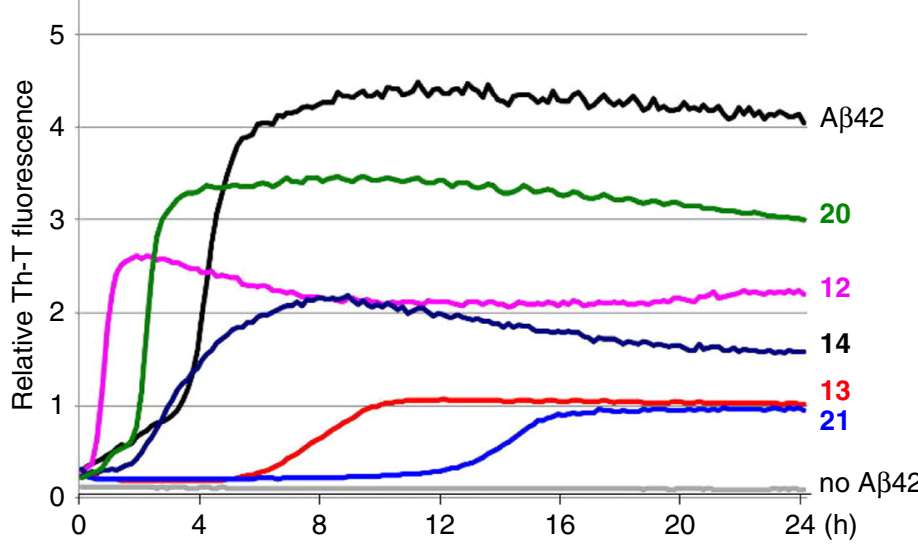

C
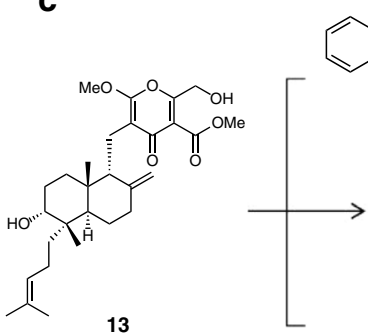

b

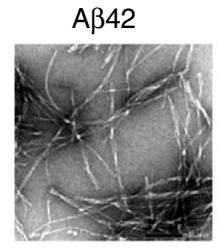

12

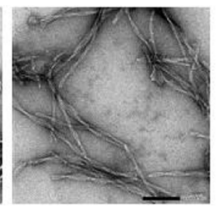

20

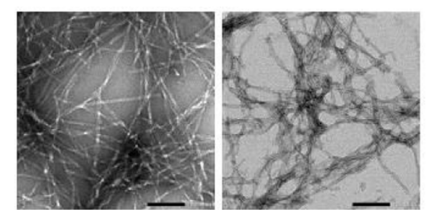

13

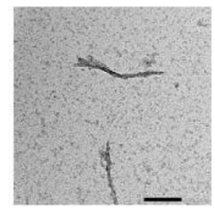

21

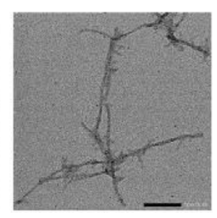

Fig. 7 Inhibitory activity of $\mathbf{A} \boldsymbol{\beta}$ aggregation. a Selected results of the Th-T assay $(n=3)$. $\mathbf{b}$ TEM analysis of typical amyloid fibrils formed by A $\beta 42$. Scale bar $=100 \mathrm{~nm}$. Representative micrographs are selected from at least six micrographs taken in one grid. $\mathbf{c}$ Hypothetical reaction mechanism of $\mathbf{1 3}$ and

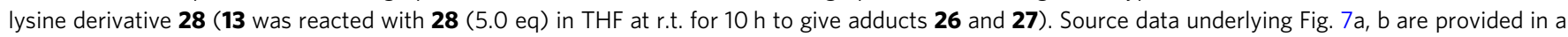
Source Data file.

did not react with the primary thiol in the cysteine derivative, the hydroxyl group in the serine derivative or the guanidine group in the arginine derivative (Supplementary Fig. 53 and Supplementary Note 32). Thus, we successfully discovered that DDPs markedly inhibited $A \beta$ aggregation in the nucleation phase via a lysine-selective binding motif.

\section{Discussion}

Herein, we report the advantage of the synthetic biology approach based on heterologous biosynthesis coupled with genome mining for rationally expanding the chemical space of biologically active natural products. In this study, we focused on fungal DDPs as a natural product family including a privileged structure and aimed to produce a diverse set of DDPs. Genome mining revealed putative gene clusters for DDPs distributed in five fungal genera and bioinformatics analyses were performed to draw the five treelike DDP biosynthetic pathways. Stepwise reconstruction of all the pathways in $A$. oryzae allowed us to make transformants corresponding to all the intermediates and end products in the pathways, resulting in the isolation of all the DDPs produced in the native DDP pathways. From their structures, we determined the function of all the modification enzymes in the pathways. Subsequently, we designed four extended pathways by using the five native pathways as tool for combinatorial biosynthesis. This combinatorial biosynthesis enabled us to access non-natural analogues that equipped further modifications than those of compounds produced via native pathways. Finally, we achieved heterologous biosynthesis of 22 DDPs, including 15 new analogues, which were included intermediates, end products, shunt products and additionally modified analogues in $A$. oryzae.

We then screened the DDP-focused library with various biological activity assays. DDPs produced in this study shared same skeleton and showed similar antiproliferative activities against cancer cells and inhibition of mitochondrial complex III each other. However, interestingly, small structure differences in each DDP gave unique functions of markedly reducing CSC-like populations in MCF-7 bulk cells (11, 12 and 22), potent inhibitory activity of an insect innate immune system, the IMD pathway (21), potent paralysing activity against adult Drosophila (5), selectively inhibited HIV proliferation (20), and A $\beta$ aggregation in the nucleation phase through trapping lysine residue on highly modified $\gamma$-pyrone motif (13 and 21). Notably, compounds produced via extended pathways indeed showed unique biological activities. Thus, this study showcases the capability of combinatorial synthetic biology in acceleration of drug discovery.

In the post-genomic era, a synthetic biology approach is undoubtedly one of the most powerful methods to achieve not only the generation of natural products from gene resources but also the rational expansion of bioactive natural product chemical space. The gene resources available in the method are rapidly increasing; therefore, the method would infinitely expand the chemical diversity of natural products and their analogues. In addition, the method solves the supply issues and permits natural products to be subjected to enough biological evaluations. When the method becomes advanced and widely used, natural products will be easier available for drug discovery and definitely increase the opportunity to develop natural products as drug seeds ${ }^{68}$.

\section{Methods}

General methods. Polymerase chain reaction (PCR) was performed using a TaKaRa PCR Thermal Cycler Dice ${ }^{\circledR}$ Gradient (Takara Bio) and Thermal Cycler LifeECO (Nippon Genetics). Oligonucleotide primers for PCR were purchased from Hokkaido System Science Co., Ltd. (Hokkaido, Japan) and listed in Supplementary Table 4. Analytical and preparative TLC was performed on silica gel 60 $\mathrm{F}_{254}$ (Merck) and RP-18 $\mathrm{F}_{254} \mathrm{~S}$ (Merck). Column chromatography and flash chromatography were carried out on silica gel $60 \mathrm{~N}(100-210 \mu \mathrm{m}$, Kanto Chemical) and silica gel $60 \mathrm{~N}(40-50 \mu \mathrm{m}$, Kanto Chemical), respectively. NMR spectra were 
recorded on a Burker AVANCE III spectrometer. Chemical shifts for ${ }^{1} \mathrm{H}$ and ${ }^{13} \mathrm{C}$ NMR are given in parts per million $(\delta)$ relative to tetramethylsilane $\left(\delta_{\mathrm{H}} 0.00\right)$ and residual solvent signals $\left(\delta_{\mathrm{C}} 77.0\right)$ for $\mathrm{CDCl}_{3}$ as internal standard. Reverse phase HPLC analysis was performed on HITACHI LaChrom Elite series equipped with L-2130 pump, L-2200 autosampler, L-2455 Diode Array Detector, and D-2000 system manager. LC-MS analysis was performed on HITACHI Chromaster series equipped with 5110 pump, 5430 Diode Array Detector, 5610 MS Detector, MSD system manager. High-resolution mass spectra were measured on a Thermo Fischer Scientific Exactive Mass spectrometer. UV spectra were recorded on a JASCO-V-730 spectrophotometer. IR spectra were recorded on JASCO-FT/IR 4200 spectrometer. Optical rotations were recorded on JASCO-P-1030.

\section{Genome mining and bio-informatics analyses of DDP pathways. Draft genome} sequence of $A$. sacchari Kumo-3 was obtained in previous study (Supplementary Data 1) ${ }^{69}$. Draft genome sequences of F. graminearum PH-1, M. phaseolina MS6, C. higginsianum IMI349063 and M. anisopliae E6 were obtained from National Center for Biotechnology Information (NCBI). Genome mining was performed by Protein BLAST search against $A$. sacchari Kumo-3 genome sequence and the NCBI non-redundant database and 2ndFind program (http://biosyn.nih.go.jp/2ndfind/). The results of bioinformatics analyses are shown in Supplementary Tables 1-3.

Fugal strains used as genomic DNA donor. Arthrinium sacchari (strain Kumo-3) was isolated from a spider previously ${ }^{69}$. Genomic DNA of Fusarium graminearum 50218 was obtained from the Medical Mycology Research Center, Chiba University (Chiba, Japan). Macrophomina phaseolina NBRC 7317 and Metarhizium anisopliae NBRC 103233 were obtained from the Biological Resource Center, National Institute of Technology and Evaluation (Chiba, Japan). Colletotrichum higginsianum MAFF 305635 was obtained from the Genetic Resource Center, National Agriculture and Food Research Organization (Ibaraki, Japan).

Heterologous host. Escherichia coli DH5a (Competent quick, TOYOBO) was used for cloning experiments. Aspergillus oryzae NSAR1 $1^{47}$, a quadruple auxotrophic mutant (niaD ${ }^{-}, s C^{-}, \triangle \arg B, a d e A^{-}$) was used as the host for fungal expression.

Preparation of fungal genomic DNA. A. sacchari Kumo-3, M. phaseolina NBRC 7317, M. anisopliae NBRC 103233, and C. higginsianum MAFF 305635 were cultivated on Potato dextrose agar (PDA) (2.4\% Potato dextrose broth (Difco), $1.5 \%$ agar). The spores and mycelium from the plate were inoculated into $60 \mathrm{~mL}$ of Potato dextrose medium (2.4\% Potato dextrose broth). After several days cultivation at $30^{\circ} \mathrm{C}$ (reciprocal shaking), the mycelia of each strain were collected by filtration, washed with water, and frozen at $-80^{\circ} \mathrm{C}$. The frozen mycelium was ground to fine powder, suspended in TE buffer ( $\mathrm{pH} 8.0$ ), and equal volume of lytic buffer ( $2 \%$ SDS, $0.1 \mathrm{M} \mathrm{NaCl}, 10 \mathrm{mM}$ EDTA, $50 \mathrm{mM}$ Tris-HCl) was added. After incubation at room temperature for $5 \mathrm{~min}$, the supernatant was extracted with phenol: chloroform: isoamyl alcohol (25:24:1) solution ( $\mathrm{pH}$ 7.9). After ethanol precipitation, the isolated DNA was dissolved in TE buffer ( $\mathrm{pH} 8.0$ ) and stored at $-20^{\circ} \mathrm{C}$ before use.

General methods for DNA engineering experiments. PCR was performed with PrimeSTAR $^{\circledast}$ Max DNA Polymerase (Takara Bio) unless otherwise noted. PCR products and linearized plasmids were purified with QIAEX $^{\circledR}$ II Gel Extraction Kit (QIAGEN). DNA cloning experiments were performed using E. coli DH5a (Competent Quick, ТОYОВО) following standard techniques and recombinant plasmids were extracted with GenElute ${ }^{\mathrm{TM}}$ Plasmid Miniprep Kit (Sigma-Aldrich) from E. coli cells.

Construction of fungal expression plasmids. The genes in $d p f g, d p m p, d p c h$, $d p a s$, and dpma clusters were amplified by PCR from genomic DNA of F. graminearum 50218, M. phaseolina NBRC 7317, C. higginsianum MAFF 305635, A. sacchari Kumo-3, and M. anisopliae NBRC 103233 as templates by using gene specific primers listed in Supplementary Table 4, respectively. The full-length genes in each cluster were purified and inserted into restriction site (Asp718 or NotI) of the pUARA2, pUADEA2, pUSCA2, or pUPTRA2 (Supplementary Fig. 3 and Supplementary Note 1) to yield fungal expression plasmid. For construction of one of two genes containing plasmids, each gene was ligated with Asp718 or/and NotIdigested pUARA2, pUADEA2, pUSCA2, or pUPTRA2 vectors using In-Fusion ${ }^{\circledast}$ HD Cloning Kit (Takara Bio) (Supplementary Fig. 4a). For construction of three or four genes containing plasmids, two-gene containing DNA fragments were amplified by PCR from the corresponding plasmids with two genes and ligated with the linearized vector fragment(s) as described above (Supplementary Fig. $4 \mathrm{~b}$ ). All the overexpression plasmid vectors made in this study are listed Supplementary Table 5 .

Transformation of $\boldsymbol{A}$. oryzae. Transformation of A. oryzae was performed using the protoplast-polyethylene glycol (PEG) method as follows: A. oryzae host strains were cultivated on agar plate (PDA supplemented with $0.05 \% \mathrm{~L}$-arginine and $0.05 \%$ adenine for NSAR1, CD agar supplemented with appropriate nutrients and chemicals (Supplementary Table 6) for transformants). The spores and mycelia of the host strain were inoculated into $60 \mathrm{~mL}$ of Czapek-Dox -Casamino acids medium (3.5\% Czapek-Dox broth (Difco), 0.5\% Casamino acids vitamin assay (Difco, when ptrA was used as a selectable marker) or Casamino acid (Difco, when ptrA was not used as a selectable marker), $0.01 \%$ adenine (for adenine auxotrophic strains)). After 20 hour cultivation at $30^{\circ} \mathrm{C}$ (reciprocal shaking), mycelium was collected by filtration and washed with water. The mycelium was incubated with $10 \mathrm{~mL}$ protoplasting buffer $\left(5 \mathrm{mg} \mathrm{mL}{ }^{-1}\right.$ Yatalase (Takara Bio), $1 \mathrm{mg} \mathrm{mL}^{-1}$ Cellulase Onozuka R-10 (SERVA Electrophoresis $\mathrm{GmbH}), 0.8 \mathrm{M} \mathrm{NaCl}, 10 \mathrm{mM}$ phosphate buffer ( $\mathrm{pH}$ 6.0)). After 3 -h incubation at $30^{\circ} \mathrm{C}$, undigested mycelia was removed by filtration with Miracloth (Merck), and the protoplasts were collected by centrifugation at $1300 \times g$ for $5 \mathrm{~min}$. The protoplasts were washed with $0.8 \mathrm{M}$ $\mathrm{NaCl}$, resuspended in solution I $(0.8 \mathrm{M} \mathrm{NaCl}, 10 \mathrm{mM} \mathrm{CaCl}, 10 \mathrm{mM}$ Tris- $\mathrm{HCl}(\mathrm{pH}$ 8.0)) to obtain $2.0 \times 10^{8}$ cells $\mathrm{mL}^{-1}$. 0.2 Volumes of solution II (40\% PEG4000, 50 $\mathrm{mM} \mathrm{CaCl}, 50 \mathrm{mM}$ Tris- $\mathrm{HCl}(\mathrm{pH} 8.0)$ ) was added with gentle mixing, and then $200 \mu \mathrm{L}$ of this protoplast suspension was combined with $10-20 \mu \mathrm{g}$ of plasmid DNA $(<20 \mu \mathrm{L})$. After $40 \mathrm{~min}$ incubation on ice, $1 \mathrm{~mL}$ of solution II was added to the aliquot with gentle mixing. After another $15 \mathrm{~min}$ of incubation at room temperature, $20 \mathrm{~mL}$ of solution I was added to the mixture and the protoplasts were collected by centrifugation at $2000 \mathrm{rpm}$ for $5 \mathrm{~min}$. The protoplasts were resuspended in $600 \mu \mathrm{L}$ of solution I, and the suspension was combined with molten softtop agar (3.5\% Czapek-Dox broth, $0.8 \mathrm{M} \mathrm{NaCl}, 0.6 \%$ agar) supplemented with appropriate nutrients (Supplementary Table 6). Subsequently, the protoplasts were plated on CD agar plates (3.5\% Czapek-Dox broth, $0.8 \mathrm{M} \mathrm{NaCl}, 1.5 \%$ agarose) with appropriate nutrients (Supplementary Table 6). After 3-7 days incubation at $30^{\circ} \mathrm{C}$, the resultant cells (transformats) were transferred and subcultured on $\mathrm{CD}$ agar plates with appropriate nutrients (Supplementary Table 6).

Reconstitution of the DDP pathways. The DDP biosynthetic genes were stepwisely introduced in A. oryzae NSAR1 using expression plasmids containing the aforementioned genes to construct all the transformants expressed the native DDP pathways (Supplementary Figs. 5, 6, 11, 16, 20, 24, 28 and Supplementary Notes 2, 7, 9, 11, 13), shunt pathway (Supplementary Fig. 30 and Supplementary Notes 15, 16,17) and extended pathways (Supplementary Figs. 34, 39 and Supplementary Notes 19, 21)

Gene integration analysis of each $\boldsymbol{A}$. oryzae transformant. We obtained about four to ten transformants per one-transformation experiments. Thus, we checked gene insertion of all the transformant by PCR. Target gene integration into the $A$. oryzae transformants were confirmed by PCR with the genomic DNA template and primers in Supplementary Table 4. Genomic DNA of the transformant was extracted as follows; small pieces of mycelium of the A. oryzae strain were collected from an agar plate after several days growth at $30^{\circ} \mathrm{C}$ and incubated at $95^{\circ} \mathrm{C}$ in $\mathrm{TE}$ $(\mathrm{pH}$ 8.0) buffer for $10 \mathrm{~min}$. The genomic DNA solution was diluted with water to prepare the PCR template at appropriate concentration.

Cultivation and metabolite analysis of $\boldsymbol{A}$. oryzae transformants. All the transformant that pass the gene integration check were cultivated and analysed their metabolite profiles by reverse phase HPLC and LC-MS analyses. Each transformant was inoculated into $60 \mathrm{~mL}$ of CPS medium (3.5\% Czapek-Dox broth, $0.3 \%$ casein peptone (Nacalai Tesque), $0.3 \%$ meat peptone (Nacalai Tesque), $0.3 \%$ soy peptone, $2 \%$ soluble starch (Nacalai Tesque), $1 \%$ maltose monohydrate (Nacalai Tesque), $0.01 \%$ adenine (for adenine auxotrophic strains)) or $1 / 2$ CPS medium $(1.75 \%$ Czapek-Dox broth, $0.15 \%$ casein peptone (Nacalai Tesque), $0.15 \%$ meat peptone (Nacalai Tesque), $0.15 \%$ soy peptone, $1 \%$ soluble starch (Nacalai Tesque), $0.5 \%$ maltose monohydrate (Nacalai Tesque), $0.01 \%$ adenine (for adenine auxotrophic strains). About 1 week (6-10 days) after cultivation at $30^{\circ} \mathrm{C}, 150 \mathrm{rpm}$, the culture media and mycelium were separated by filtration. The culture media $(3 \mathrm{~mL})$ was extracted with EtOAc $(2 \mathrm{~mL})$, and the organic layer was concentrated in vacuo. The residue was then dissolved in $150 \mu \mathrm{L}$ of $\mathrm{MeOH}$ to prepare sample for reverse phase HPLC and LC-MS analysis. The freeze-dried mycelium $(20 \mathrm{mg})$ was extracted with $\mathrm{MeOH}(1 \mathrm{~mL})$, and the extract was concentrated in vacuo. The residue was then dissolved in $100 \mu \mathrm{L}$ of $\mathrm{MeOH}$ to prepare sample for HPLC and LC-MS analysis. For the HPLC and LC-MS analysis, $10 \mu \mathrm{L}$ of the samples were used. The HPLC analysis was performed on COSMOSIL $5 \mathrm{C}_{18}$ Packed Column $(4.6 \mathrm{~mm}$ I.D. $\times 150 \mathrm{~mm}$, Nacalai Tesque) with acetonitrile and water containing $0.01 \%$ trifluoroacetic acid (0-1.5 min: 20:80, 1.5-11.5 min: a linear gradient from 20:80 to 100:0, 11.5-20 min: 100:0) at a flow rate of $1.0 \mathrm{~mL} \mathrm{~min}^{-1}$. The LC-MS analysis was performed on COSMOSIL $5 \mathrm{C}_{18}$ Packed Column (4.6 mm I.D. $\times 150 \mathrm{~mm}$, Nacalai Tesque) with acetonitrile containing $0.1 \%$ formic acid and water containing $0.1 \%$ formic acid (0-2 min: 20:80, 2-12 min: a linear gradient from 20:80 to 100:0, 12-20 min: 100:0) at a flow rate of $1.0 \mathrm{~mL} \mathrm{~min}^{-1}$ using positive mode electro spray ionization.

Isolation and structure determination of each compound. To isolate DDPs produced by the transformants, we picked up the transformants that produced target DDPs the most and performed scaling up cultivation of the transformant. Each A. oryzae transformant was cultivated in 1/2 CPS medium (1.75\% CzapekDox broth, $0.15 \%$ casein peptone (Nacalai Tesque), $0.15 \%$ meat peptone (Nacalai Tesque), $0.15 \%$ soy peptone, $1 \%$ soluble starch (Nacalai Tesque), $0.5 \%$ maltose monohydrate (Nacalai Tesque), $0.01 \%$ adenine (for adenine auxotrophic strains) 
for 1 week at $30{ }^{\circ} \mathrm{C}$, and the culture media and mycelium were separated by filtration. The culture media was extracted with EtOAc two times, and the organic layer were combined and concentrated in vacuo to give crude extract. The freezedried mycelium was extracted with EtOAc $(20 \% \mathrm{MeOH})$, and the extract was concentrated in vacuo. The mycelia extract was then dissolved in $\mathrm{MeOH}(0.1 \%$ water), washed with hexane three times, concentrated in vacuo to give crude extract. Each of the crude extract of culture and mycelium was fractionated by silica gel column chromatography, and subjected to further purification by flash chromatography and preparative TLC $\left(\mathrm{SiO}_{2}\right.$ or ODS). All the DDP structures were fully determined by spectral analyses (Supplementary Figs. 8-10, 14, 15, 18, 19, 22, 23, $26,27,32,33,37,38,41,42,52,54-77$, Supplementary Tables 7-18, 25 and Supplementary Notes $3-6,8,10,12,14,18,20,22,31$ ).

Evaluation of biological activities of DDP-focused library. Purified DDPs wa dissolved in DMSO (Specially Prepared Reagent, Nuclease and Protease tested, NACALAI, 09659-14) to prepare $5 \mathrm{mM}$ solutions. The Each DDP solution $(5 \mathrm{mM})$ was used for antiproliferative effects on cancer cell lines (Supplementary Notes 23, $24,25,26$ ), biological activity against Drosophila (Supplementary Note 27), antiHIV assay (Supplementary Note 28) and screening of amyloid Ab42 aggregation inhibitors (Supplementary Notes 29).

Reporting summary. Further information on research design is available in the Nature Research Reporting Summary linked to this article.

\section{Data availability}

The data supporting the findings of this work are available within the paper and its Supplementary Information files. A reporting summary for this Article is available as a Supplementary Information file. The data sets generated and analyzed during this study are available from the corresponding author upon request. The sequences data of $d p a s A$ $F$ and Dpas A-F are provided as a Supplementary Data 1. The source data underlying Figs. 5a, c, d, 6a, b, 7a, b, Supplementary Figs. 44, 46a, 47, and Supplementary Tables 20, 23 , and 24 are provided as a Source Data file.

Received: 18 October 2019; Accepted: 19 March 2020;

Published online: 14 April 2020

\section{References}

1. Koehn, F. E. \& Carter, G. T. The evolving role of natural products in drug discovery. Nat. Rev. Drug Discov. 4, 206-220 (2005).

2. Newman, D. J. \& Cragg, G. M. Natural products as source of new drugs from 1981 to 2014. J. Nat. Prod. 79, 629-661 (2016).

3. Matsuda, Y. \& Abe, I. Biosynthesis of fungal meroterpenoids. Nat. Prod. Rep. 33, 26-53 (2016).

4. Masschelein, J., Jenner, M. \& Challis, G. L. Antibiotics from Gram-negative bacteria: a comprehensive overview and selected biosynthetic highlights. Nat. Prod. Rep. 34, 712-783 (2017).

5. Kellenberger, E., Hofmann, A. \& Quinn, R. J. Similar interactions of natural products with biosynthetic enzymes and therapeutic targets could explain why nature produces such a large proportion of existing drugs. Nat. Prod. Rep. 28, 1483-1492 (2011).

6. Rosén, J., Gottfries, J., Muresan, S., Backlund, A. \& Oprea, T. I. Novel chemical space exploration via natural products. J. Med. Chem. 52, 1953-1962 (2009).

7. Clardy, J. \& Walsh, C. Lessons from natural molecules. Nature 432, 829-837 (2004).

8. Over, B. et al. Natural-product-derived fragments for fragment-based ligand discovery. Nat. Chem. 5, 21-28 (2013).

9. Maier, M. E. Design and synthesis of analogues of natural products. Org. Biomol. Chem. 13, 5302-5343 (2015).

10. Robles, O. \& Romo, D. Chemo- and site-selective derivatizations of natural products enabling biological studies. Nat. Prod. Rep. 31, 318-334 (2014).

11. Harvey, A. L., Edrada-Ebel, R. \& Quinn, R. J. The re-emergence of natural products for drug discovery in the genomics era. Nat. Rev. Drug Discov. 14, 111-129 (2015).

12. Jørgensen, L. et al. 14-step synthesis of (+)-ingenol from (+)-3-carene. Science 341, 878-882 (2013).

13. Kuroda, Y. et al. Isolation, synthesis and bioactivity studies of phomactin terpenoids. Nat. Chem. 10, 938-945 (2018).

14. Llabani, E. et al. Diverse compounds from pleuromutilin lead to a thioredoxin inhibitor and inducer of ferroptosis. Nat. Chem. 11, 521-532 (2019).

15. Balthaser, B. R., Maloney, M. C., Beeler, A. B., Porco, J. A. \& Snyder, J. K. Remodeling of the natural product fumagillol employing a reaction discovery approach. Nat. Chem. 3, 969-973 (2011).

16. Huigens, R. W. et al. A ring-distortion strategy to construct stereochemically complex and structurally diverse compounds from natural products. Nat. Chem. 5, 195-202 (2013).
17. Asai, T. et al. Use of a biosynthetic intermediate to explore the chemical diversity of pseudo-natural fungal polyketides. Nat. Chem. 7, 737-743 (2015).

18. Keller, N. P., Turner, G. \& Bennett, J. W. Fungal secondary metabolism from biochemistry to genomics. Nat. Rev. Microbiol. 3, 937-947 (2005).

19. Sanchez, J. F., Somoza, A. D., Keller, N. P. \& Wang, C. C. C. Advances in Aspergillus secondary metabolite research in the post-genomic era. Nat. Prod. Rep. 29, 351-371 (2012).

20. Hertweck, C. Hidden biosynthetic treasures brought to light. Nat. Chem. Biol. 5, 450-452 (2009).

21. Li, L. et al. Genome mining and assembly-line biosynthesis of the UCS1025A pyrrolizidinone family of fungal alkaloids. J. Am. Chem. Soc. 140, 2067-2071 (2016).

22. Fujii, R. et al. H. Total biosynthesis of diterpene aphidicolin, a specific inhibitor of DNA polymerase $\alpha$ : heterologous expression of four biosynthetic genes in Aspergillus oryzae. Biosci. Biotechnol. Biochem. 75, 1813-1817 (2011).

23. Tagami, K. et al. Reconstitution of biosynthetic machinery for indolediterpene paxilline in Aspergillus oryzae. J. Am. Chem. Soc. 135, 1260-1263 (2013).

24. Matsuda, Y., Wakimoto, T., Mori, T., Awakawa, T. \& Abe, I. Complete biosynthetic pathway of anditomin: nature's sophisticated synthetic route to a complex fungal meroterpenoid. J. Am. Chem. Soc. 136, 15326-15336 (2014).

25. Itoh, T. et al. Reconstitution of a fungal meroterpenoid biosynthesis reveals the involvement of a novel family of terpene cyclases. Nat. Chem. 2, 858-864 (2010).

26. Morishita, Y., Zhang, H., Taniguchi, T., Mori, K. \& Asai, T. The discovery of fungal polyene macrolides via a postgenomic approach reveals a polyketide macrocyclization by trans-acting thioesterase in fungi. Org. Lett. 21, 4788-4792 (2019).

27. Kaneko, A., Morishita, Y., Tsukada, K., Taniguchi, T. \& Asai, T. Genomebased discovery of alkylresorcinols from a cricket-associated fungus, Penicillium soppi, that displays an unusual polyketide biosynthetic machinery. Org. Biomol. Chem. 17, 5239-5243 (2019).

28. Harvey, C. J. B. et al. HEx: a heterologous expression platform for the discovery of fungal natural products. Sci. Adv. 4, eaar5459 (2018).

29. Liu, T., Chiang, Y. M., Somoza, A. D., Oakley, B. R. \& Wang, C. C. C. Engineering of an "unnatural" natural product by swapping polyketide synthase domains in Aspergillus nidulans. J. Am. Chem. Soc. 133, 13314-13316 (2011).

30. Xu, Y. et al. Diversity-oriented combinatorial biosynthesis of benzenediol lactone scaffolds by subunit shuffling of fungal polyketide synthases. Proc. Natl Acad. Sci. USA 111, 12354-12359 (2014).

31. Awakawa, T. et al. Reprogramming of the antimycin NRPS-PKS assembly lines inspired by gene evolution. Nat. Commun. 9, 3534 (2018).

32. Yan, F. et al. Synthetic biology approaches and combinatorial biosynthesis towards heterologous lipopeptide production. Chem. Sci. 9, 7510-7519 (2018).

33. Gupta, S. et al. Viridoxins A and B: novel toxins from the fungus Metarhizium flavoviride. J. Org. Chem. 58, 1062-1067 (1993).

34. Engel, B., Erkel, G., Anke, T. \& Sterner, O. Sesquicillin, an inhibitor of glucocorticoid mediated signal transduction. J. Antibiot. 51, 518-521 (1998).

35. Uchida, R. et al. New sesquicillins, insecticidal antibiotics produced by Albophoma sp. FKI-1778. J. Antibiot. 58, 397-404 (2005).

36. Lai, K. et al. Integrated compound profiling screens identify the mitochondrial electron transport chain as the molecular target of the natural products manassantin, sesquicillin, and arctigenin. ACS Chem. Biol. 8, 257-267 (2013).

37. Kikuchi, H. et al. New diterpene pyrone-type compounds, metarhizins A and $\mathrm{B}$, isolated from entomopathogenic fungus, Metarhizium flavoviride and their inhibitory effects on cellular proliferation. Tetrahedron 65, 469 (2009).

38. Lee, J. C., Lobkovsky, E., Pliam, N. B., Strobel, G. \& Clardy, J. Subglutinols A and B: immunosuppressive compounds from the endophytic fungus Fusarium subglutinans. J. Org. Chem. 60, 7076-7077 (1995).

39. Lee, W. G. et al. Immunosuppressive effects of subglutinol derivatives. ChemMedChem 7, 218-222 (2012).

40. Cimmino, A. et al. Higginsianins A and B, two diterpenoid a-pyrones produced by Colletotrichum higginsianum, with in vitro cytostatic activity. $J$. Nat. Prod. 79, 116-125 (2016).

41. Gohbara, M. et al. Structures of colletotrichin and colletotrichin B, phytotoxic metabolites from Colletotrichum nicotianae. Agric. Biol. Chem. 42, 1037-1043 (1978).

42. Zhang, F. \& Danishefsky, S. J. An efficient stereoselective total synthesis of DL-sesquicillin, a glucocorticoid antagonist. Angew. Chem. Int. Ed. 41, 1434-1437 (2002).

43. Kim, H. et al. Stereoselective synthesis and osteogenic activity of subglutinols A and B. J. Am. Chem. Soc. 131, 3192-3194 (2009).

44. Oguchi, T., Watanabe, K., Ohkubo, K., Abe, H. \& Katou, T. Enantioselective total synthesis of (-)-subglutinols A and B: potential immunosuppressive agents isolated from a microorganism. Chem. Eur. J. 15, 2826-2845 (2009).

45. Merchant, R. R. et al. Divergent synthesis of pyrone diterpenes via radical cross coupling. J. Am. Chem. Soc. 140, 7462-7465 (2018). 
46. Kato, $\mathrm{H}$. et al. New natural products isolated from Metarhizium robertsii ARSEF 23 by chemical screening and identification of the gene cluster through engineered biosynthesis in Aspergillus nidulans A1145. J. Antibiot. 69, 561-566 (2016).

47. Jin, F. J., Maruyama, J., Juvvadi, P. R., Arioka, M. \& Kitamoto, K. Development of a novel quadruple auxotrophic host transformation system by $\operatorname{argB}$ gene disruption using adeA gene and exploiting adenine auxotrophy in Aspergillus oryzae. FEMS Microbiol. Lett. 239, 79-85 (2004).

48. Matsuda, Y., Awakawa, T. \& Abe, I. Reconstituted biosynthesis of fungal meroterpenoid andrastin A. Tetrahedron 69, 8199-8204 (2013).

49. Ohtani, I., Kusumi, T., Kashman, Y. \& Kakisawa, H. High-field FT NMR application of Mosher's method. The absolute configurations of marine terpenoids. J. Am. Chem. Soc. 113, 4092-4096 (1991)

50. Dan, S. et al. An integrated database of chemosensitivity to 55 anticancer drugs and gene expression profiles of 39 human cancer cell lines. Cancer Res. 62, 1139-1147 (2002).

51. Kitamura, K., Itoh, H., Ssakurai, K., Dan, S. \& Inoue, M. Target Identification of Yaku'amide B and its two distinct activities against mitochondrial F0F1ATP synthase. J. Am. Chem. Soc. 140, 12189-12199 (2018).

52. Lytle, N. K., Barber, A. G. \& Reya, T. Stem cell fate in cancer growth, progression and therapy resistance. Nat. Rev. Cancer 18, 669-680 (2018).

53. Bai, X., Ni, J., Beretov, J., Graham, P. \& Li, Y. Cancer stem cell in breast cancer therapeutic resistance. Cancer Treat. Rev. 69, 152-163 (2018).

54. Park, J. H., Chung, S., Matsuo, Y. \& Nakamura, Y. Development of small molecular compounds targeting cancer stem cells. Medchemcomm 8, 73-80 (2016).

55. Hirata, N. et al. Sphingosine-1-phosphate promotes expansion of cancer stem cells via S1PR3 by a ligand-independent Notch activation. Nat. Commun. 5, 4806 (2014).

56. Chen, J. et al. Inhibition of cancer stem cell like cells by a synthetic retinoid. Nat. Commun. 9, 1406 (2018).

57. Lemaitre, B. \& Hoffmann, J. The host defense of Drosophila melanogaster. Annu. Rev. Immunol. 25, 697-743 (2007).

58. Kanoh, H. et al. Genome-wide RNAi screening implicates the E3 ubiquitin ligase Sherpa in mediating innate immune signaling by Toll in Drosophila adults. Sci. Signal. 8, ra107 (2015).

59. Manaka, J. et al. Draper-mediated and phosphatidylserine-independent phagocytosis of apoptotic cells by Drosophila Hemocytes/Macrophages. J. Biol. Chem. 279, 48466-48476 (2004)

60. Nonaka, S. et al. Characterization of Spz5 as a novel ligand for Drosophila Toll-1 receptor. Biochem. Biophys. Res. Commun. 506, 510-515 (2018).

61. Kanoh, H. et al. Ex vivo genome-wide RNAi screening of the Drosophila Toll signaling pathway elicited by a larva-derived tissue extract. Biochem. Biophys. Res. Commun. 467, 400-406 (2015).

62. Kajiwara, K., Kodama, E. \& Matsuoka, M. A novel colorimetric assay for CXCR4 and CCR5 tropic human immunodeficiency viruses. Antivir. Chem. Chemother. 17, 215-223 (2006).

63. Haass, C. \& Selkoe, D. J. Soluble protein oligomers in neurodegeneration: lessons from the Alzheimer's amyloid beta-peptide. Nat. Rev. Mol. Cell Biol. 8, 101-112 (2007).

64. Hasegawa, K., Yamaguchi, I., Omata, S., Gejyo, F. \& Naiki, H. Interaction between A beta(1-42) and A beta(1-40) in Alzheimer's beta-amyloid fibril formation in vitro. Biochemistry 38, 15514-15521 (1999).

65. Serio, T. R. et al. Nucleated conformational conversion and the replication of conformational information by a prion determinant. Science 289, 1317-1321 (2000).

66. Murakami, K. et al. Monoclonal antibody against the turn of the 42-residue amyloid $\beta$-protein at positions and 23. ACS Chem. Neurosci. 17, 747-756 (2010).

67. Hanaki, M. et al. Mechanistic analyses of the suppression of amyloid $\beta 42$ aggregation by apomorphine. Bioorg. Med. Chem. 26, 1538-1546 (2018).

68. Shen, B. A new golden age of natural products drug discovery. Cell 163, 1297-1300 (2015).

69. Morishita, Y. et al. Use of plant hormones to activate silent polyketide biosynthetic pathways in Arthrinium sacchari, a fungus isolated from a spider. Org. Biomol. Chem. 17, 780-784 (2019).

\section{Acknowledgements}

The authors thank Prof. K. Gomi (Tohoku University) and Prof. K. Kitamoto (The University of Tokyo) for providing the expression vectors and the fungal strain. The authors thank Prof. T. Kuroda (Hiroshima University) and Prof. D. Hagiwara (Tsukuba University) for preliminary screening of anti-bacterial and anti-fungal compounds. The JFCR39 cancer cell line panel assays were kindly performed by Molecular Profiling Committee, Grant-in-Aid for Scientific Research on Innovative Areas "Platform of Advanced Animal Model Support (AdAMS)” from The Ministry of Education, Culture, Sports, Science and Technology, Japan (KAKENHI 16H06276 to S.D.). This work was supported by JSPS KAKENHI (grant no. 17H05055, 17H05428, 18K19388 and 19 H04642 to T.A., 16H06194 to K.M. and 17H04107 to Y.K.) from Japan Society for the Promotion of Science (JSPS)), the Naito Foundation and Astellas Foundation for Research on Metabolic Disorders to T.A.

\section{Author contributions}

T.A. conceived this study, and designed the experiments. T.A. and K.T. analysed all the DDP clusters and performed all the synthetic biology experiments and characterized all the DDPs. T.A. and K.T. also performed chemical reactions. S.S. and A.K. contributed to construct $A$. oryzae transformants and analysed their metabolite profiles by HPLC analyses. K.M. and K.I. designed, performed and analysed the assay for amyloid A $\beta 42$ aggregation inhibition. M.M. and H.M. designed, performed and analyzed assay for inhibitory activities on mitochondrial respiratory complex. S.D. designed, performed and analysed the assay for antiproliferative effects across the panel of JFCR39 cancer cell lines. A.H., E.S. and T.K. designed, performed and analysed the assay using Drosophila. K.K., H.H. and E.N.K. designed, performed and analysed the assay for anti-viral activity. N.H. and Y.K. designed, performed and analysed the assay for anti-CSC activity. T.A. wrote this manuscript with input from all authors. All authors have given their approval of the final version of the manuscript.

\section{Competing interests}

The authors declare no competing interests.

\section{Additional information}

Supplementary information is available for this paper at https://doi.org/10.1038/s41467020-15664-4.

Correspondence and requests for materials should be addressed to T.A.

Peer review information Nature Communications thanks Ren Xiang Tan, and the other, anonymous, reviewer(s) for their contribution to the peer review of this work. Peer reviewer reports are available.

Reprints and permission information is available at http://www.nature.com/reprints

Publisher's note Springer Nature remains neutral with regard to jurisdictional claims in published maps and institutional affiliations.

Open Access This article is licensed under a Creative Commons Attribution 4.0 International License, which permits use, sharing, adaptation, distribution and reproduction in any medium or format, as long as you give appropriate credit to the original author(s) and the source, provide a link to the Creative Commons license, and indicate if changes were made. The images or other third party material in this article are included in the article's Creative Commons license, unless indicated otherwise in a credit line to the material. If material is not included in the article's Creative Commons license and your intended use is not permitted by statutory regulation or exceeds the permitted use, you will need to obtain permission directly from the copyright holder. To view a copy of this license, visit http://creativecommons.org/ licenses/by/4.0/

(C) The Author(s) 2020 